\title{
Eighty Nine
}

National Cancer Institute

\section{Source}

National Cancer Institute. Eighty Nine. NCI Thesaurus. Code C105789.

A natural number greater than eighty-eight and less than ninety and the quantity that it denotes. 